\title{
INVESTIGATION OF A COST-EFFECTIVE FINITE DIFFERENCE SCHEME FOR THE TRANSPORT EQUATION ${ }^{1}$
}

\author{
D.G. SLUGIN, A.V. POPOV \\ Moscow State University \\ Moscow, Russia \\ E-mail: slugindm@mtu-net.ru; popovav@mech.math.msu.su
}

Received May 28, 2003; revised September 15, 2003

\begin{abstract}
The transport equation for three-dimensional flow of a viscous gas is considered. An implicit finite difference scheme is constructed for approximating the transport equation. The error estimation is proved. The main part of the analysis is done for the first differential approximation of the proposed finite difference scheme, but the results are also valid in the fully discrete case.
\end{abstract}

Key words: transport equation, viscous gas, three-dimensional case, implicit finite difference scheme

\section{INTRODUCTION}

Many laws of the nature can be expressed by partial differential equations, which can be a part of complex systems of the equations describing many actual problems. The equation of continuity is one of the examples. There are many explicit methods for solving quasilinear and first-order systems (see $[1 ; 2 ; 4 ; 7])$. However Courant's theorem (domains of dependence [2]) imposes a limitation on mesh steps and such restrictions don't always correspond to properties of the problem. For implicit difference schemes, which do not have a limitation on mesh steps, there are many difficulties in development of algorithms of finding a discrete solution in the case of multidimensional

\footnotetext{
${ }^{1}$ This work was made with support of RAN programme "Modern problems of theoretical mathematics", project "Optimization of computational algorithms for problems of mathematical physics" and Russian Science Foundation for Basic Research, project N 0201-00490.
} 
problems.

In the given work by using the factorization in space method we develop an implicit finite-difference method for solving three dimensional first order hyperbolic problems.

For simplicity of presentation we will limit our analysis to the simple transport equation:

$$
\frac{\partial u}{\partial t}+\sum_{i=1}^{3} \frac{\partial\left(a_{i} u\right)}{\partial x_{i}}=0
$$

where $u=u(t, \mathbf{x})$ is defined in the domain

$$
Q=[0, T] \times \Omega, \quad \Omega=\left[0, X_{1}\right] \times\left[0, X_{2}\right] \times\left[0, X_{3}\right] .
$$

The equation contains known functions $a_{k}=a_{k}(t, \mathbf{x})(k=1,2,3)$, which satisfy the following boundary conditions

$$
a_{1}(t, \mathbf{x})=a_{2}(t, \mathbf{x})=a_{3}(t, \mathbf{x})=0, \quad(t, \mathbf{x}) \in[0, T] \times \partial \Omega,
$$

where $\partial \Omega$ is a boundary of $\Omega$. The following initial condition is formulated:

$$
\left.u\right|_{t=0}=u_{0}(\mathbf{x}), \quad \mathbf{x} \in \Omega .
$$

The examined method has been proposed in works $[5 ; 6]$ for two-dimensional non-stationary flow of a viscous gas. We will generalize this result in threedimensional case. Using this scheme some problems of convection and flow of a viscous gas (in three dimensional case) were computed with good accuracy.

\section{NOTATIONS}

Let us introduce in the domain $Q=[0, T] \times \Omega$ a mesh $Q_{\tau \mathbf{h}}=\bar{\omega}_{\tau} \times \bar{\omega}_{\mathbf{h}}$ :

$$
\begin{aligned}
& \bar{\omega}_{\tau}=\{j \tau: j=0, \ldots, M\}, \tau M=T, \\
& \bar{\omega}_{\mathbf{h}}=\left\{\left(i_{1} h_{1}, i_{2} h_{2}, i_{3} h_{3}\right): i_{j}=0, \ldots, N_{j}, h_{j} N_{j}=X_{j} j=1,2,3\right\} .
\end{aligned}
$$

Below we use the following notations for subsets of $\bar{\omega}_{\mathbf{h}}$ :

$$
\begin{array}{ll}
\gamma_{k}=\left\{\mathbf{i h} \mid i_{k}=N_{k}\right\}, & \gamma_{-k}=\left\{\mathbf{i h} \mid i_{k}=0\right\}, \\
\gamma_{\mathbf{h}}=\bigcup_{k=1}^{3}\left(\gamma_{k} \cup \gamma_{-k}\right), & \omega_{\mathbf{h}}=\bar{\omega}_{\mathbf{h}} \backslash \gamma_{\mathbf{h}} .
\end{array}
$$


The value of a mesh function $g$ at a node $\left(j, i_{1}, i_{2}, i_{3}\right)$ is denoted by $g_{i_{1}, i_{2}, i_{3}}^{j}$. The omission of indices of the function means that $g=g_{i_{1}, i_{2}, i_{3}}^{j}$. It will also be useful to define the following notations for the values of functions at nodes neighbouring to a node $\left(j, i_{1}, i_{2}, i_{3}\right)$

$g_{i_{1}, i_{2}, i_{3}}^{j+1}=\hat{g}, \quad g_{i_{1} \pm 1, i_{2}, i_{3}}^{j}=g^{\left( \pm 1_{1}\right)}, \quad g_{i_{1}, i_{2} \pm 1, i_{3}}^{j}=g^{\left( \pm 1_{2}\right)}, \quad g_{i_{1}, i_{2}, i_{3} \pm 1}^{j}=g^{\left( \pm 1_{3}\right)}$.

The mean value of a mesh function at two neighbouring nodes will be written in the following form

$$
g_{s_{k},\left(i_{1}, i_{2}, i_{3}\right)}=\frac{1}{2}\left(g_{i_{1}, i_{2}, i_{3}}^{\left(+1_{k}\right)}+g_{i_{1}, i_{2}, i_{3}}\right), \quad g_{\bar{s}_{k},\left(i_{1}, i_{2}, i_{3}\right)}=\frac{1}{2}\left(g_{i_{1}, i_{2}, i_{3}}+g_{i_{1}, i_{2}, i_{3}}^{\left(-1_{k}\right)}\right) .
$$

The following notations are used for difference operators

$$
\begin{aligned}
& g_{t}=\frac{\hat{g}-g}{\tau}, \quad \partial_{x_{k}} g \equiv g_{x_{k}}=\frac{g^{\left(+1_{k}\right)}-g}{h_{k}}, \\
& \partial_{\bar{x}_{k}} g \equiv g_{\bar{x}_{k}}=\frac{g-g^{\left(-1_{k}\right)}}{h_{k}}, \quad \partial_{x_{k}}^{\circ} g \equiv g_{x_{k}}=\frac{g^{\left(+1_{k}\right)}-g^{\left(-1_{k}\right)}}{2 h_{k}} .
\end{aligned}
$$

Let us introduce the discrete norm $\|g\|$ (a mesh analogue of the $L_{2}$ norm)

$$
\|g\|=\sqrt{\sum_{\bar{\omega}_{h}} h_{1} h_{2} h_{3} g_{i_{1}, i_{2}, i_{3}}^{2} .}
$$

The semi-norm $|g|_{1}$ is defined by

$$
|g|_{1}=\sqrt{\sum_{k=1}^{3} \sum_{\bar{\omega}_{h} \backslash \gamma_{k}} h_{1} h_{2} h_{3} g_{x_{k}}^{2}} .
$$

Finally we define the norm $\|g\|_{1}$ (a mesh analogue of $W_{2}^{1}$ norm)

$$
\|g\|_{1}=\sqrt{\|g\|^{2}+|g|_{1}^{2}}
$$

The following variant of the Gronwall lemma (see [3]) will be used to prove the theorem of numerical approximation error estimation.

Lemma 2.1. Let values $Y_{0}, Y_{1}, \ldots, Y_{N}$ satisfy the following inequality

$$
Y_{n} \leq Y_{n-1}+C \tau\left(Y_{n-1}+Y_{n}\right)+\tau b_{n}, \quad n=1, \ldots, N .
$$

Besides it is known, that

$$
b_{n} \geq 0, \text { for } n=1, \ldots, N, \tau<\tau_{0}, \tau_{0} C \leq 1 / 2, \tau N=T .
$$


Then these values obey the estimation

$$
\max _{n=1, \ldots, N} \sqrt{Y_{n}} \leq e^{C T} \sqrt{Y_{0}+2 \tau \sum_{n=1}^{N} b_{n}} .
$$

\section{DIFFERENCE SCHEME}

Let the mesh function $v$ be an approximation of the exact solution of (1.1)(1.2). We consider the following difference scheme:

$$
\prod_{k=1}^{3}\left(E+\tau A_{k}^{1}\left(a_{k}\right)+\nu \tau^{2} A_{k}^{2}(\mathbf{a})\right) \hat{v}=v+\tau b(v, \mathbf{a}) .
$$

As the initial condition we use the projection of function $u_{0}$ (see, (1.2)) onto the mesh $\omega_{h}$ :

$$
v_{i_{1}, i_{2}, i_{3}}^{0}=u_{0}\left(i_{1} h_{1}, i_{2} h_{2}, i_{3} h_{3}\right) .
$$

Here $\nu$ is a positive constant, which will be defined later. Operators $A_{k}^{1}$ approximate the part of the difference divergence operator containing partial derivative with respect to $x_{k}$ :

$$
A_{k}^{1}\left(a_{k}\right) v= \begin{cases}\frac{1}{2}\left(\left(a_{k} v\right)_{\bar{x}_{k}}^{o}+a_{k} v_{x_{k}}^{\circ}\right), \quad \mathbf{x}_{\mathbf{i}} \in \bar{\omega}_{\mathbf{h}} \backslash\left(\gamma_{k} \cup \gamma_{-k}\right), \\ \frac{1}{2}\left(a_{k} v\right)_{x_{k}}, \quad \mathbf{x}_{\mathbf{i}} \in \gamma_{-k}, \\ \frac{1}{2}\left(a_{k} v\right)_{\bar{x}_{k}}, \quad \mathbf{x}_{\mathbf{i}} \in \gamma_{k} .\end{cases}
$$

Operators $A_{k}^{2}$ are added as the stabilizers of the scheme

$$
A_{k}^{2}(\mathbf{a}) v= \begin{cases}-\left(\Phi_{k} v_{x_{k}}\right)_{\bar{x}_{k}}, & \mathbf{x}_{\mathbf{i}} \in \bar{\omega}_{\mathbf{h}} \backslash\left(\gamma_{k} \cup \gamma_{-k}\right), \\ -2 h_{k}^{-1} \Phi_{k} v_{x_{k}}, & \mathbf{x}_{\mathbf{i}} \in \gamma_{-k}, \\ 2 h_{k}^{-1} \Phi_{k}^{\left(-1_{k}\right)} v_{\bar{x}_{k}}, & \mathbf{x}_{\mathbf{i}} \in \gamma_{k} .\end{cases}
$$

The mesh functions $\Phi_{1}, \Phi_{2}$ and $\Phi_{3}$ are given by

$$
\Phi_{1}=|\mathbf{a}|_{x_{1} x_{2} \bar{x}_{2} x_{3} \bar{x}_{3}}^{2}, \quad \Phi_{2}=|\mathbf{a}|_{x_{1} \bar{x}_{1} x_{2} x_{3} \bar{x}_{3}}^{2}, \quad \Phi_{3}=|\mathbf{a}|_{x_{1} \bar{x}_{1} x_{2} \bar{x}_{2} x_{3}}^{2},
$$

where $|\mathbf{a}|^{2}=\sum_{i=1}^{3} a_{i}^{2}$. The function $|\mathbf{a}|^{2}$ is continued beyond the boundary of the mesh in the odd way. 
The function $b(v, \mathbf{a})$ is defined by

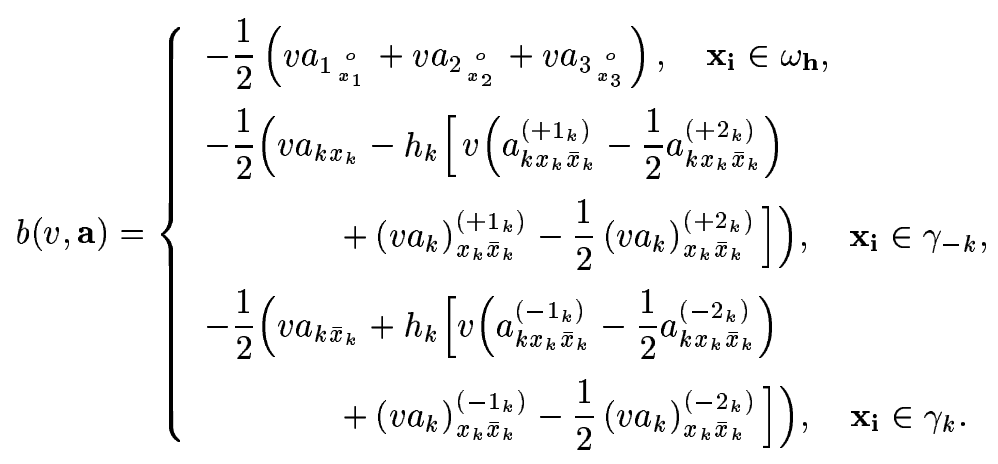

Theorem 3.1. The difference scheme (3.1) - (3.2) has a unique solution.

Proof. The solution of the problem (3.1) - (3.2) is reduced to the computation of the mesh function $\hat{v}$ at the time step $j+1$ using the already known functions $v$ from the previous time step $j$. In order to determine the unknown function $\hat{v}$ we obtain from (3.1) the system of linear algebraic equations

$$
A_{1} A_{2} A_{3} \hat{v}=b,
$$

where $A_{k}(k=1,2,3)$ is the square matrix of the size $\left(N_{1}+1\right)\left(N_{2}+1\right)\left(N_{3}+1\right) \times$ $\left(N_{1}+1\right)\left(N_{2}+1\right)\left(N_{3}+1\right)$. The matrix $A_{k}$ is block-diagonal and the size of each block is $\left(N_{k}+1\right) \times\left(N_{k}+1\right) . A_{k}$ is a tridiagonal matrix, which is a sum of three matrices: the first matrix is diagonal, the second matrix is skew-symmetric and the third one is symmetric and nonnegative. As a consequence of this structure, each block is nonsingular, that the whole matrix $A_{k}$ is nonsingular. Thus the difference scheme always has a unique solution, which can be found by the sweep method.

\section{ANALYSIS OF THE SCHEME}

We will denote by $\varphi$ a local truncation error of the finite difference scheme (3.1)-(3.2). Then $\|\varphi\|_{1}$ is of order $O\left(\tau+\mathbf{h}^{2}\right)$.

Let us introduce a global error function $w$, which is a difference between the solution of (3.1) - (3.2) and the projection of the exact solution $u$ onto the mesh $Q_{\tau \mathbf{h}}$ :

$$
w=v-u .
$$

The strict convergence analysis of the finite difference scheme (3.1) - (3.2) requires many technical manipulations, therefore for simplicity we will make our analysis for a corresponding differential problem. All estimations are also valid in the discrete case. 
We use below the following notations and differential operators:

$$
\begin{gathered}
u_{t}=\frac{\partial u}{\partial t}, \quad u_{x_{k}}=\frac{\partial u}{\partial x_{k}}, \quad(k=1,2,3) \\
(u, v)=\int_{\Omega} u v d x, \quad\|u\|=\sqrt{\int_{\Omega} u^{2} d x} \\
|u|_{1}=\sqrt{\int_{\Omega} \sum_{k=1}^{3} u_{x_{k}}^{2} d x, \quad\|u\|_{1}}=\sqrt{\|u\|^{2}+|u|_{1}^{2}} \\
B_{k}^{1}\left(a_{k}\right) w=\frac{1}{2}\left(\frac{\partial\left(a_{k} w\right)}{\partial x_{k}}+a_{k} \frac{\partial w}{\partial x_{k}}\right) \\
B_{k}^{2}(\mathbf{a}) w=-\frac{\partial}{\partial x_{k}}\left(|\mathbf{a}|^{2} \frac{\partial(w)}{\partial x_{k}}\right), k=1 \ldots 3 \\
b(\mathbf{a}, w)=\frac{1}{2} \sum_{k=1}^{3} w \frac{\partial a_{k}}{\partial x_{k}}
\end{gathered}
$$

Let us substitute $w$ into the scheme $(3.1)-(3.2)$, then we obtain the following differential problem:

$$
\begin{aligned}
w_{t} & +\left(B_{1}^{1}+B_{2}^{1}+B_{3}^{1}\right) w-b(\mathbf{a}, w) \\
& +\tau\left(B_{1}^{1}\left(a_{1}\right) B_{2}^{1}\left(a_{2}\right)+B_{1}^{1}\left(a_{1}\right) B_{3}^{1}\left(a_{3}\right) B_{2}^{1}\left(a_{2}\right) B_{3}^{1}\left(a_{3}\right)\right) w \\
& +\nu \tau\left(B_{1}^{2}(\mathbf{a})+B_{2}^{2}(\mathbf{a})+B_{3}^{2}(\mathbf{a})\right) w+\tau^{2} B_{1}^{1}\left(a_{1}\right) B_{2}^{1}\left(a_{2}\right) B_{3}^{1}\left(a_{3}\right) w \\
& +\nu \tau^{2}\left[B_{1}^{1}\left(a_{1}\right) B_{2}^{2}(\mathbf{a})+B_{1}^{1}\left(a_{1}\right) B_{3}^{2}(\mathbf{a})+B_{1}^{2}(\mathbf{a}) B_{2}^{1}\left(a_{1}\right)+B_{2}^{1}\left(a_{1}\right) B_{3}^{2}(\mathbf{a})\right. \\
& \left.+B_{1}^{2}(\mathbf{a}) B_{3}^{1}\left(a_{3}\right)+B_{2}^{2}(\mathbf{a}) B_{3}^{1}\left(a_{3}\right)\right] w+\nu \tau^{3}\left[B_{1}^{1}\left(a_{1}\right) B_{2}^{1}\left(a_{2}\right) B_{3}^{2}(\mathbf{a})\right. \\
& \left.+B_{1}^{1}\left(a_{1}\right) B_{2}^{2}(\mathbf{a}) B_{3}^{1}\left(a_{3}\right)+B_{1}^{2}(\mathbf{a}) B_{2}^{1}\left(a_{2}\right) B_{3}^{1}\left(a_{3}\right)\right] w+\nu^{2} \tau^{3}\left[B_{1}^{2}(\mathbf{a}) B_{2}^{2}(\mathbf{a})\right. \\
& \left.+B_{1}^{2}(\mathbf{a}) B_{3}^{2}(\mathbf{a})+B_{2}^{2}(\mathbf{a}) B_{3}^{2}(\mathbf{a})\right] w+\nu^{2} \tau^{4}\left[B_{1}^{1}\left(a_{1}\right) B_{2}^{2}(\mathbf{a}) B_{3}^{2}(\mathbf{a})\right. \\
& \left.+B_{1}^{2}(\mathbf{a}) B_{2}^{1}\left(a_{2}\right) B_{3}^{2}(\mathbf{a})+B_{1}^{2}(\mathbf{a}) B_{2}^{2}(\mathbf{a}) B_{3}^{1}\left(a_{3}\right)\right] w \\
& +\nu^{3} \tau^{5} B_{1}^{2}(\mathbf{a}) B_{2}^{2}(\mathbf{a}) B_{3}^{2}(\mathbf{a}) w=\varphi \\
w & \\
t=0 & =0
\end{aligned}
$$

Multiplying the equation (4.1) by $w$ and integrating it over $\Omega$, we obtain the following equalities

$$
\left(w, w_{t}\right)=\frac{\left(\|w\|^{2}\right)_{t}}{2}
$$


Investigation of a finite difference scheme for the transport equation 253

$$
\begin{aligned}
\left(w,\left(B_{1}^{1}+B_{2}^{1}+B_{3}^{1}\right) w\right)=\left(w, \frac{1}{2}\left(a_{1} w\right)_{x_{1}}+\frac{1}{2}\left(a_{2} w\right)_{x_{2}}\right. \\
\left.+\frac{1}{2}\left(a_{3} w\right)_{x_{3}}+\frac{a_{1}}{2} w_{x_{1}}+\frac{a_{2}}{2} w_{x_{2}}+\frac{a_{3}}{2} w_{x_{3}}\right)=0 .
\end{aligned}
$$

Using the $\varepsilon$-inequality and the other well-known inequalities we estimate each term of the obtained equality (here $C$ are constants, which depend only on the size of the domain $Q$ and the norm of the exact solution of (1.1)-(1.2)):

$$
\begin{aligned}
& |(w, b(\mathbf{a}, w))|=\left|\left(w, \frac{w}{2} a_{1_{x_{1}}}+\frac{w}{2} a_{2_{x_{2}}}+\frac{w}{2} a_{3_{x_{3}}}\right)\right| \leq C|| w \|^{2}, \\
& |(w, \varphi)| \leq \frac{\|w\|^{2}+\|\varphi\|^{2}}{2} \\
& \tau\left|\left(w,\left(B_{1}^{1}\left(a_{1}\right) B_{2}^{1}\left(a_{2}\right)+B_{1}^{1}\left(a_{1}\right) B_{3}^{1}\left(a_{3}\right)+B_{2}^{1}\left(a_{2}\right) B_{3}^{1}\left(a_{3}\right)\right) w\right)\right| \\
& =\frac{\tau}{4} \mid\left(w,\left(a_{1}\left(a_{2} w\right)_{x_{2}}\right)_{x_{1}}+a_{1}\left(a_{2} w\right)_{x_{2} x_{1}}+a_{1}\left(a_{2} w_{x_{2}}\right)_{x_{1}}+\left(a_{1} a_{2} w_{x_{2}}\right)_{x_{1}}\right. \\
& +\left(a_{1}\left(a_{3} w\right)_{x_{3}}\right)_{x_{1}}+a_{1}\left(a_{3} w\right)_{x_{3} x_{1}}+a_{1}\left(a_{3} w_{x_{3}}\right)_{x_{1}}+\left(a_{1} a_{3} w_{x_{3}}\right)_{x_{1}} \\
& \left.+\left(a_{2}\left(a_{3} w\right)_{x_{3}}\right)_{x_{2}}+a_{2}\left(a_{3} w\right)_{x_{3} x_{2}}+a_{2}\left(a_{3} w_{x_{3}}\right)_{x_{2}}+\left(a_{2} a_{3} w_{x_{3}}\right)_{x_{2}}\right) \mid \\
& \leq \tau(1+\tau C)\left(\left\||\mathbf{a}| w_{x_{1}}\right\|^{2}+\left\||\mathbf{a}| w_{x_{2}}\right\|^{2}+\left\||\mathbf{a}| w_{x_{3}}\right\|^{2}\right)+\left.C\|w\|\right|^{2}, \\
& \nu \tau\left(w,\left(B_{1}^{2}(\mathbf{a})+B_{2}^{2}(\mathbf{a})+B_{3}^{2}(\mathbf{a})\right) w\right) \\
& =-\nu \tau\left(w,\left(|\mathbf{a}|^{2} w_{x_{1}}\right)_{x_{1}}+\left(|\mathbf{a}|^{2} w_{x_{2}}\right)_{x_{2}}+\left(|\mathbf{a}|^{2} w_{x_{3}}\right)_{x_{3}}\right) \\
& =\tau \nu\left(|||\mathbf{a}| w_{x_{1}}||^{2}+\left\||\mathbf{a}| w_{x_{2}}\right\|^{2}+\|\left.|\mathbf{a}| w_{x_{3}}\right|^{2}\right) \text {, } \\
& \tau^{2}\left|\left(w, B_{1}^{1}\left(a_{1}\right) B_{2}^{1}\left(a_{2}\right) B_{3}^{1}\left(a_{3}\right) w\right)\right| \\
& +\nu \tau^{2} \mid\left(w,\left[B_{1}^{1}\left(a_{1}\right) B_{2}^{2}(\mathbf{a})+B_{1}^{1}\left(a_{1}\right) B_{3}^{2}(\mathbf{a})+B_{1}^{2}(\mathbf{a}) B_{2}^{1}\left(a_{1}\right)\right.\right. \\
& \left.\left.+B_{2}^{1}\left(a_{1}\right) B_{3}^{2}(\mathbf{a})+B_{1}^{2}(\mathbf{a}) B_{3}^{1}\left(a_{3}\right)+B_{2}^{2}(\mathbf{a}) B_{3}^{1}\left(a_{3}\right)\right] w\right) \\
& \leq \tau(1+\tau C)\left(\left\||\mathbf{a}| w_{x_{1}}\right\|^{2}+\left\||\mathbf{a}| w_{x_{2}}\right\|^{2}+\left\||\mathbf{a}| w_{x_{3}}\right\|^{2}\right)+\frac{\tau^{3}}{2}\left(1+\nu^{2}\right) \\
& \times\left(\left\||\mathbf{a}|^{2} w_{x_{1} x_{2}}\right\|^{2}+\left\||\mathbf{a}|^{2} w_{x_{2} x_{3}}\right\|^{2}+\left\||\mathbf{a}|^{2} w_{x_{1} x_{3}}\right\|^{2}\right)+\tau C\|w\|^{2}, \\
& \nu \tau^{3} \mid\left(w,\left[B_{1}^{1}\left(a_{1}\right) B_{2}^{1}\left(a_{2}\right) B_{3}^{2}(\mathbf{a})\right.\right. \\
& \left.\left.+B_{1}^{1}\left(a_{1}\right) B_{2}^{2}(\mathbf{a}) B_{3}^{1}\left(a_{3}\right)+B_{1}^{2}(\mathbf{a}) B_{2}^{1}\left(a_{2}\right) B_{3}^{1}\left(a_{3}\right)\right] w\right) \mid \\
& \left.\left.\leq \nu \tau^{3}(1+\tau C)\left(\left\||\mathbf{a}|^{2} w_{x_{1} x_{2}}\right\|^{2}\right)+\left\||\mathbf{a}|^{2} w_{x_{2} x_{3}}\right\|^{2}\right)+\left\||\mathbf{a}|^{2} w_{x_{1} x_{3}}\right\|^{2}\right) \\
& \left.\left.+C \tau^{2}\left(\left\||\mathbf{a}| w_{x_{1}}\right\|^{2}\right)+\left\||\mathbf{a}| w_{x_{2}}\right\|^{2}\right)+\left\||\mathbf{a}| w_{x_{3}}\right\|^{2}\right),
\end{aligned}
$$




$$
\begin{gathered}
\nu^{2} \tau^{3}\left(w,\left[B_{1}^{2}(\mathbf{a}) B_{2}^{2}(\mathbf{a})+B_{1}^{2}(\mathbf{a}) B_{3}^{2}(\mathbf{a})+B_{2}^{2}(\mathbf{a}) B_{3}^{2}(\mathbf{a})\right] w\right) \\
\geq \nu^{2} \tau^{3}(1-\tau C)\left(\left\||\mathbf{a}|^{2} w_{x_{1} x_{2}}\right\|^{2}+\left\||\mathbf{a}|^{2} w_{x_{2} x_{3}}\right\|^{2}+\left\||\mathbf{a}|^{2} w_{x_{1} x_{3}}\right\|^{2}\right) \\
-C \tau^{3}\left(\left\||\mathbf{a}| w_{x_{1}}\right\|^{2}+\left\||\mathbf{a}| w_{x_{2}}\right\|^{2}+\left\||\mathbf{a}| w_{x_{3}}\right\|^{2}\right)-C \tau|| w \|^{2} \\
\nu^{2} \tau^{4}\left|\left(w,\left[B_{1}^{1}\left(a_{1}\right) B_{2}^{2}(\mathbf{a}) B_{3}^{2}(\mathbf{a})+B_{1}^{2}(\mathbf{a}) B_{2}^{1}\left(a_{2}\right) B_{3}^{2}(\mathbf{a})+B_{1}^{2}(\mathbf{a}) B_{2}^{2}(\mathbf{a}) B_{3}^{1}\left(a_{3}\right)\right] w\right)\right| \\
\leq \frac{\nu^{2} \tau^{5}}{2}\left\||\mathbf{a}|^{3} w_{x_{1} x_{2} x_{3}}\right\|^{2}+\nu \tau^{3}(1+\tau C)\left(\left\||\mathbf{a}|^{2} w_{x_{1} x_{2}}\right\|^{2}+\left\||\mathbf{a}|^{2} w_{x_{2} x_{3}}\right\|^{2}\right. \\
\left.+\left\||\mathbf{a}|^{2} w_{x_{1} x_{3}}\right\|^{2}\right)+C \tau^{3}\left(\left\||\mathbf{a}| w_{x_{1}}\right\|^{2}+\left\||\mathbf{a}| w_{x_{2}}\right\|^{2}+\||\mathbf{a}| w_{x_{3}}||^{2}\right), \\
\nu^{3} \tau^{5}\left(w, B_{1}^{2}(\mathbf{a}) B_{2}^{2}(\mathbf{a}) B_{3}^{2}(\mathbf{a}) w\right) \geq \nu^{3} \tau^{5}(1-\tau C)\left\||\mathbf{a}|^{3} w_{x_{1} x_{2} x_{3}}\right\|^{2} \\
-C \tau^{4}\left(\left\||\mathbf{a}|^{2} w_{x_{1} x_{2}}\right\|^{2}+\left\||\mathbf{a}|^{2} w_{x_{2} x_{3}}\right\|^{2}+\left\||\mathbf{a}|^{2} w_{x_{1} x_{3}}\right\|^{2}\right) \\
-C \tau^{5}\left(\left\||\mathbf{a}| w_{x_{1}}\right\|^{2}+\left\||\mathbf{a}| w_{x_{2}}\right\|^{2}+\left\||\mathbf{a}| w_{x_{3}}\right\|^{2}\right) .
\end{gathered}
$$

Substituting all these inequalities into Eq. (4.1) we obtain

$$
\begin{aligned}
\frac{\left(\|\left. w\right|^{2}\right)_{t}}{2} & \left.\left.+\tau(\nu-3-\tau C)\left(\left\||\mathbf{a}| w_{x_{1}}\right\|^{2}\right)+\left\||\mathbf{a}| w_{x_{2}}\right\|^{2}\right)+\left\||\mathbf{a}| w_{x_{3}}\right\|^{2}\right) \\
& \left.+\frac{\tau^{2}}{2}\left(\nu^{2}-4 \nu-1-\tau C\right)\left(\left\||\mathbf{a}|^{2} w_{x_{1} x_{2}}\right\|^{2}\right)+\left\||\mathbf{a}|^{2} w_{x_{2} x_{3}}\right\|^{2}\right) \\
& \left.+\left\||\mathbf{a}|^{2} w_{x_{1} x_{3}}\right\|^{2}\right)+\nu^{3} \frac{\tau^{5}}{2}(1-\tau C)\left\||\mathbf{a}|^{3} w_{x_{1} x_{2} x_{3}}\right\|^{2} \leq C\|w\|^{2}+\frac{\|\varphi\|^{2}}{2} .
\end{aligned}
$$

Next let us differentiate the equation (4.1) with respect to $x_{1}$, multiply it by $w_{x_{1}}$ and integrate over $\Omega$. Then we estimate each part of the equality and obtain the following results:

$$
\begin{aligned}
& \left(w_{x_{1}}, w_{t x_{1}}\right)=\frac{\left(\left|w_{x_{1}}\right|_{1}^{2}\right)_{t}}{2}, \quad\left|\left(w_{x_{1}}, \varphi_{x_{1}}\right)\right| \leq \frac{\left\|w_{x_{1}}\right\|^{2}+\left\|\varphi_{x_{1}}\right\|^{2}}{2}, \\
& \left|\left(w_{x_{1}},\left(B_{1}^{1}+B_{2}^{1}+B_{3}^{1}\right)_{x_{1}} w\right)\right| \leq C\|w\|_{1}^{2}, \quad\left|\left(w_{x_{1}}, b(\mathbf{a}, w)_{x_{1}}\right)\right| \leq C\|w\|_{1}^{2}, \\
\tau & \left|\left(w_{x_{1}},\left(B_{1}^{1}\left(a_{1}\right) B_{2}^{1}\left(a_{2}\right)+B_{1}^{1}\left(a_{1}\right) B_{3}^{1}\left(a_{3}\right)+B_{2}^{1}\left(a_{2}\right) B_{3}^{1}\left(a_{3}\right)\right)_{x_{1}} w\right)\right| \\
& +\nu \tau\left(w_{x_{1}},\left(B_{1}^{2}(\mathbf{a})+B_{2}^{2}(\mathbf{a})+B_{3}^{2}(\mathbf{a})\right)_{x_{1}} w\right) \geq \tau(\nu-1-\tau C)\left(\left\||\mathbf{a}| w_{x_{1} x_{1}}\right\|^{2}\right. \\
& \left.+\left\||\mathbf{a}| w_{x_{1} x_{2}}\right\|^{2}+\left\||\mathbf{a}| w_{x_{1} x_{3}}\right\|^{2}\right)-C\|w\|_{1}^{2},
\end{aligned}
$$


Investigation of a finite difference scheme for the transport equation 255

$$
\begin{aligned}
& \tau^{2}\left|\left(w_{x_{1}},\left[B_{1}^{1}\left(a_{1}\right) B_{2}^{1}\left(a_{2}\right) B_{3}^{1}\left(a_{3}\right)\right]_{x_{1}} w\right)\right|++\nu \tau^{2} \mid\left(w_{x_{1}},\left[B_{1}^{1}\left(a_{1}\right) B_{2}^{2}(\mathbf{a})\right.\right. \\
& +B_{1}^{1}\left(a_{1}\right) B_{3}^{2}(\mathbf{a})+B_{1}^{2}(\mathbf{a}) B_{2}^{1}\left(a_{1}\right)+B_{2}^{1}\left(a_{1}\right) B_{3}^{2}(\mathbf{a})+B_{1}^{2}(\mathbf{a}) B_{3}^{1}\left(a_{3}\right) \\
& \left.\left.+B_{2}^{2}(\mathbf{a}) B_{3}^{1}\left(a_{3}\right)\right]_{x_{1}} w\right) \mid \leq \tau(1+\tau C)\left(\left\||\mathbf{a}| w_{x_{1} x_{1}}\right\|^{2}+\left\||\mathbf{a}| w_{x_{1} x_{2}}\right\|^{2}\right. \\
& \left.+|||\mathbf{a}| w_{x_{1} x_{3}} \|^{2}\right)+\frac{\tau^{3}}{2}\left(1+\nu^{2}\right)\left(\left.|||\mathbf{a}|^{2} w_{x_{1} x_{1} x_{2}}\left\|^{2}+\right\||\mathbf{a}|^{2} w_{x_{1} x_{2} x_{3}}\right|^{2}\right. \\
& \left.+\left.|||\mathbf{a}|^{2} w_{x_{1} x_{1} x_{3}}\right|^{2}\right)+\tau C|| w \|_{1}^{2}, \\
& \nu \tau^{3} \mid\left(w_{x_{1}},\left[B_{1}^{1}\left(a_{1}\right) B_{2}^{1}\left(a_{2}\right) B_{3}^{2}(\mathbf{a})+B_{1}^{1}\left(a_{1}\right) B_{2}^{2}(\mathbf{a}) B_{3}^{1}\left(a_{3}\right)\right.\right. \\
& \left.\left.+B_{1}^{2}(\mathbf{a}) B_{2}^{1}\left(a_{2}\right) B_{3}^{1}\left(a_{3}\right)\right]_{x_{1}} w\right) \mid \leq \nu \tau^{3}(1+\tau C)\left(\left\||\mathbf{a}|^{2} w_{x_{1} x_{1} x_{2}}\right\|^{2}\right. \\
& \left.+\left.|||\mathbf{a}|^{2} w_{x_{1} x_{2} x_{3}}\left\|^{2}+\right\||\mathbf{a}|^{2} w_{x_{1} x_{1} x_{3}}\right|^{2}\right)++C \tau^{2}\left(\left\||\mathbf{a}| w_{x_{1} x_{1}}\right\|^{2}\right. \\
& \left.+\left\||\mathbf{a}| w_{x_{1} x_{2}}\right\|^{2}+\left\||\mathbf{a}| w_{x_{1} x_{3}}\right\|^{2}\right)+C \tau|| w||_{2}^{1}, \\
& \nu^{2} \tau^{3}\left(w_{x_{1}},\left[B_{1}^{2}(\mathbf{a}) B_{2}^{2}(\mathbf{a})+B_{1}^{2}(\mathbf{a}) B_{3}^{2}(\mathbf{a})+B_{2}^{2}(\mathbf{a}) B_{3}^{2}(\mathbf{a})\right]_{x_{1}} w\right) \geq \nu^{2} \tau^{3}(1-\tau C) \\
& \times\left(|||\mathbf{a}|^{2} w_{x_{1} x_{1} x_{2}}\left\|^{2}+|||\mathbf{a}|^{2} w_{x_{1} x_{2} x_{3}}\right\|^{2}+|||\mathbf{a}|^{2} w_{x_{1} x_{1} x_{3}} \|^{2}\right) \\
& -C \tau^{3}\left(\left\||\mathbf{a}| w_{x_{1} x_{1}}\right\|^{2}+\left\||\mathbf{a}| w_{x_{1} x_{2}}\right\|^{2}+\left\||\mathbf{a}| w_{x_{1} x_{3}}\right\|^{2}\right)-C \tau\|\mid w\|_{1}^{2}, \\
& \nu^{2} \tau^{4} \mid\left(w_{x_{1}},\left[B_{1}^{1}\left(a_{1}\right) B_{2}^{2}(\mathbf{a}) B_{3}^{2}(\mathbf{a})+B_{1}^{2}(\mathbf{a}) B_{2}^{1}\left(a_{2}\right) B_{3}^{2}(\mathbf{a})\right.\right. \\
& \left.\left.+B_{1}^{2}(\mathbf{a}) B_{2}^{2}(\mathbf{a}) B_{3}^{1}\left(a_{3}\right)\right]_{x_{1}} w\right)\left|\leq \frac{\nu^{2} \tau^{5}}{2}\left\||\mathbf{a}|^{3} w_{x_{1} x_{1} x_{2} x_{3}}\right\|^{2}+C \tau^{3}\left(\left\||\mathbf{a}| w_{x_{1} x_{1}}\right\|^{2}\right.\right. \\
& \left.+|||\mathbf{a}| w_{x_{1} x_{2}}\left\|^{2}+\right\||\mathbf{a}| w_{x_{1} x_{3}} \|^{2}\right)+\nu \tau^{3}(1+\tau C)\left(|||\mathbf{a}|^{2} w_{x_{1} x_{1} x_{2}} \|^{2}\right. \\
& \left.+|||\mathbf{a}|^{2} w_{x_{1} x_{2} x_{3}}\left\|^{2}+\right\||\mathbf{a}|^{2} w_{x_{1} x_{1} x_{3}} \|^{2}\right)+C \tau\|w\|_{1}^{2}, \\
& \nu^{3} \tau^{5}\left(w_{x_{1}},\left(B_{1}^{2}(\mathbf{a}) B_{2}^{2}(\mathbf{a}) B_{3}^{2}(\mathbf{a})\right)_{x_{1}} w\right) \geq \nu^{3} \tau^{5}(1-\tau C)\left\||\mathbf{a}|^{3} w_{x_{1} x_{1} x_{2} x_{3}}\right\|^{2} \\
& -\tau^{4} C\left(\left\||\mathbf{a}|^{2} w_{x_{1} x_{1} x_{2}}||^{2}+|||\mathbf{a}|^{2} w_{x_{1} x_{2} x_{3}}\right\|^{2}+|||\mathbf{a}|^{2} w_{x_{1} x_{1} x_{3}} \|^{2}\right) \\
& -C \tau^{5}\left(\left\||\mathbf{a}| w_{x_{1} x_{1}}\right\|^{2}+\left\||\mathbf{a}| w_{x_{1} x_{2}}\right\|^{2}+\|\left.|\mathbf{a}| w_{x_{1} x_{3}}\right|^{2}\right)-C \tau|| w \|_{1}^{2} \text {. }
\end{aligned}
$$

Using all these estimations we get the following inequality:

$$
\begin{aligned}
\frac{\left(\left\|w_{x_{1}}\right\|^{2}\right)_{t}}{2} & +\tau(\nu-3-\tau C)\left(\left\||\mathbf{a}| w_{x_{1} x_{1}}\right\|^{2}+\left\||\mathbf{a}| w_{x_{1} x_{2}}\right\|^{2}+|||\mathbf{a}| w_{x_{1} x_{3}} \|^{2}\right) \\
& +\frac{\tau^{2}}{2}\left(\nu^{2}-4 \nu-1-\tau C\right)\left(\left\||\mathbf{a}|^{2} w_{x_{1} x_{1} x_{2}}\right\|^{2}+\left\||\mathbf{a}|^{2} w_{x_{1} x_{2} x_{3}}\right\|^{2}\right.
\end{aligned}
$$




$$
\left.+\left\||\mathbf{a}|^{2} w_{x_{1} x_{1} x_{3}}\right\|^{2}\right)++\nu^{3} \frac{\tau^{5}}{2}(1-\tau C)\left\||\mathbf{a}|^{3} w_{x_{1} x_{1} x_{2} x_{3}}\right\|^{2} \leq C|| w \|_{1}^{2}+\frac{\left\|\varphi_{x_{1}}\right\|^{2}}{2} .
$$

Analogously we can get the estimations of derivatives with respect to $x_{2}$, $x_{3}$ and sum them. Finally we obtain the stability estimate:

$$
\begin{aligned}
\frac{\left(|w|_{1}^{2}\right)_{t}}{2} & +\tau(\nu-3-\tau C)\left(\left\||\mathbf{a}| w_{x_{1} x_{1}}\right\|^{2}+\left\||\mathbf{a}| w_{x_{1} x_{2}}\right\|^{2}+\left.\left\||\mathbf{a}| w_{x_{1} x_{3}}\right\|\right|^{2}\right. \\
& +\left\||\mathbf{a}| w_{x_{2} x_{2}}\right\|^{2}+\left\||\mathbf{a}| w_{x_{1} x_{2}}\right\|^{2}+\left\||\mathbf{a}| w_{x_{2} x_{3}}\right\|^{2}+\left\||\mathbf{a}| w_{x_{3} x_{3}}\right\|^{2} \\
& \left.+\left\||\mathbf{a}| w_{x_{1} x_{3}}\right\|^{2}+\left\||\mathbf{a}| w_{x_{2} x_{3}}\right\|^{2}\right)+\frac{\tau^{2}}{2}\left(\nu^{2}-4 \nu-1-\tau C\right) \\
& \times\left(3\left\||\mathbf{a}|^{2} w_{x_{1} x_{2} x_{3}}\right\|^{2}+\left\||\mathbf{a}|^{2} w_{x_{1} x_{1} x_{2}}\right\|^{2}+\left\||\mathbf{a}|^{2} w_{x_{1} x_{1} x_{3}}\right\|^{2}\right. \\
& +\left\||\mathbf{a}|^{2} w_{x_{2} x_{2} x_{1}}\right\|^{2}+\left\||\mathbf{a}|^{2} w_{x_{2} x_{2} x_{3}}\right\|^{2}+\left\||\mathbf{a}|^{2} w_{x_{3} x_{3} x_{1}}\right\|^{2} \\
& \left.+\left\||\mathbf{a}|^{2} w_{x_{3} x_{3} x_{2}}\right\|^{2}\right)+\nu^{3} \frac{\tau^{5}}{2}(1-\tau C)\left(\left\||\mathbf{a}|^{3} w_{x_{1} x_{1} x_{2} x_{3}}\right\|^{2}\right. \\
& \left.+\left\||\mathbf{a}|^{3} w_{x_{1} x_{2} x_{2} x_{3}}\right\|^{2}+\left\||\mathbf{a}|^{3} w_{x_{1} x_{2} x_{3} x_{3}}\right\|^{2}\right) \leq C|| w_{1} \|_{1}^{2}+\frac{|\varphi|_{1}^{2}}{2}
\end{aligned}
$$

Applying a similar approach to the finite-difference equations we obtain analogous estimates in the discrete norms $(2.1)-(2.3)$ :

$$
\begin{aligned}
\frac{\|\hat{w}\|^{2}-\|w\|^{2}}{2 \tau} & +\tau(\nu-3-\tau C)\left(\left\||\mathbf{a}| w_{x_{1}}\right\|^{2}+\left\||\mathbf{a}| w_{x_{2}}\right\|^{2}+\left\||\mathbf{a}| w_{x_{3}}\right\|^{2}\right) \\
& +\frac{\tau^{2}}{2}\left(\nu^{2}-4 \nu-1-\tau C\right)\left(\left\||\mathbf{a}|^{2} w_{x_{1} x_{2}}\right\|^{2}+\left\||\mathbf{a}|^{2} w_{x_{2} x_{3}}\right\|^{2}\right. \\
& \left.+\left\||\mathbf{a}|^{2} w_{x_{1} x_{3}}\right\|^{2}\right)++\nu^{3} \frac{\tau^{5}}{2}(1-\tau C)\left\||\mathbf{a}|^{3} w_{x_{1} x_{2} x_{3}}\right\|^{2} \\
& \leq C\|w\|^{2}+\frac{\|\varphi\|^{2}}{2}
\end{aligned}
$$

and

$$
\begin{aligned}
\frac{|\hat{w}|_{1}^{2}-|w|_{1}^{2}}{2 \tau} & +\tau(\nu-3-\tau C)\left(\left\||\mathbf{a}| w_{x_{1} x_{1}}\right\|^{2}+\left\||\mathbf{a}| w_{x_{1} x_{2}}\right\|^{2}+\left\||\mathbf{a}| w_{x_{1} x_{3}}\right\|^{2}\right. \\
& +\left\||\mathbf{a}| w_{x_{2} x_{2}}\right\|^{2}+\left\||\mathbf{a}| w_{x_{1} x_{2}}\right\|^{2}+\left\||\mathbf{a}| w_{x_{2} x_{3}}\right\|^{2}+\left\||\mathbf{a}| w_{x_{3} x_{3}}\right\|^{2} \\
& \left.+\left\||\mathbf{a}| w_{x_{1} x_{3}}\right\|^{2}\right)+\frac{\tau^{2}}{2}\left(\nu^{2}-4 \nu-1-\tau C\right)\left(3|||\mathbf{a}|^{2} w_{x_{1} x_{2} x_{3}} \|^{2}\right. \\
& +\left\||\mathbf{a}|^{2} w_{x_{1} x_{1} x_{2}}\right\|^{2}+\left\||\mathbf{a}|^{2} w_{x_{1} x_{1} x_{3}}\right\|^{2}+\left\||\mathbf{a}|^{2} w_{x_{2} x_{2} x_{1}}\right\|^{2} \\
& \left.+\left\||\mathbf{a}|^{2} w_{x_{2} x_{2} x_{3}}\right\|^{2}+\left\||\mathbf{a}|^{2} w_{x_{3} x_{3} x_{1}}\right\|^{2}+\left\||\mathbf{a}|^{2} w_{x_{3} x_{3} x_{2}}\right\|^{2}\right)
\end{aligned}
$$


Investigation of a finite difference scheme for the transport equation 257

$$
\begin{array}{r}
+\nu^{3} \frac{\tau^{5}}{2}(1-\tau C)\left(\left\||\mathbf{a}|^{3} w_{x_{1} x_{1} x_{2} x_{3}}\right\|^{2}+\left\||\mathbf{a}|^{3} w_{x_{1} x_{2} x_{2} x_{3}}\right\|^{2}\right. \\
\left.+\left\||\mathbf{a}|^{3} w_{x_{1} x_{2} x_{3} x_{3}}\right\|^{2}\right) \leq C\|w\|_{1}^{2}+\frac{|\varphi|_{1}^{2}}{2} .
\end{array}
$$

We choose parameters $\nu$ and $\tau_{\max }$ such that the following estimates hold:

$$
\nu-3-\tau C \geq 0, \quad \nu^{2}-4 \nu-1-\tau C \geq 0, \quad 1-\tau C \geq 0 .
$$

For example, these inequalities are valid if $\nu \geq 5$ and $0 \leq \tau \leq 1 / C$.

After that we sum (4.5) and (4.6), use the notation

$$
Y_{n}=\|\hat{w}\|_{1}^{2}, \quad Y_{n-1}=\|w\|_{1}^{2}
$$

and obtain the main stability inequality

$$
Y_{n} \leq Y_{n-1}+C \tau Y_{n-1}+\tau\|\varphi\|_{1}^{2}
$$

From (4.2) we have the initial condition

$$
Y_{0}=0
$$

Using the Lemma 2.1 we prove the following theorem:

Theorem 4.1. Let the following conditions are satisfied for the differential problem (1.1)-(1.2)

- there exists a unique classical solution;

- $u, a_{k} \in C^{4,2}(Q)$, where $C^{p, q}(Q)$ is a class of functions having derivatives of order $p$ with respect to $\mathbf{x}$ and order $q$ with respect to $t$.

Then the following estimate of the global error

$$
\max _{n=1, \ldots, M}\left\|u^{n}-v^{n}\right\|_{1} \leq C\left(\tau+\mathbf{h}^{2}\right)
$$

is valid for sufficiently small $\tau \leq \tau_{\max }, \mathbf{h} \leq \mathbf{h}_{\max }$, where $\tau_{\max }, \mathbf{h}_{\max }$ and $C$ depend only on the size of the domain $Q$ and on the exact solution of the differential problem.

\section{REFERENCES}

[1] N.S. Bachvalov, N.P. Zhidkov and G.M. Kobelkov. The computing methods. Nauka, 1987. 
[2] O.M. Belocherkovsky, V.A. Andruchenko and U.D. Chevelev. The dynamics of spatial vortical flows in a non-uniform atmosphere. Computing experiment. Yanys-K, 2000.

[3] E.G. Diakonov. The difference methods of solutions the boundary equations. MSU, 1971.

[4] A.G. Kulikovsky, N.V. Pogorelov and A.U. Semenov. Mathematical questions of the difference solution of hyperbolic systems of the equations. FIZMATLIT, 2001.

[5] A.V. Popov. A study of cost-effective finite difference scheme for the system of equations for two-dimensional flow of a viscous barotropic gas. Sov.J.Numer.Mat.Modelling, 5, $395-417,1990$.

[6] A.V. Popov. The implicit difference schemes for a flow of a viscous gas problem. Works of Kazan Mathematics SOCIETY, 13, 2001.

[7] A.A. Samarsky. The theory of difference schemes. Nauka, 1983.

\section{Ekonomiškų baigtinių skirtumų schemų pernešimo lygčiai tyrimas}

D. Slugin, A.Popov

Straipsnyje nagrinèjama trimačių klampių dujų tekèjimo pernešimo lygtis. Pateikta neišreikštinè baigtinių skirtumų schema aproksimuojanti šią pernešimo lygtị. İrodytas paklaidos ivertis. Pagrindinè tyrimo analizè atlikta pateiktos baigtiniu skirtumu schemos pirmos eilès diferencialiniam artiniui. Analogiški rezultatai pasilieka teisingi ir pilnai diskrečiuoju atveju. 